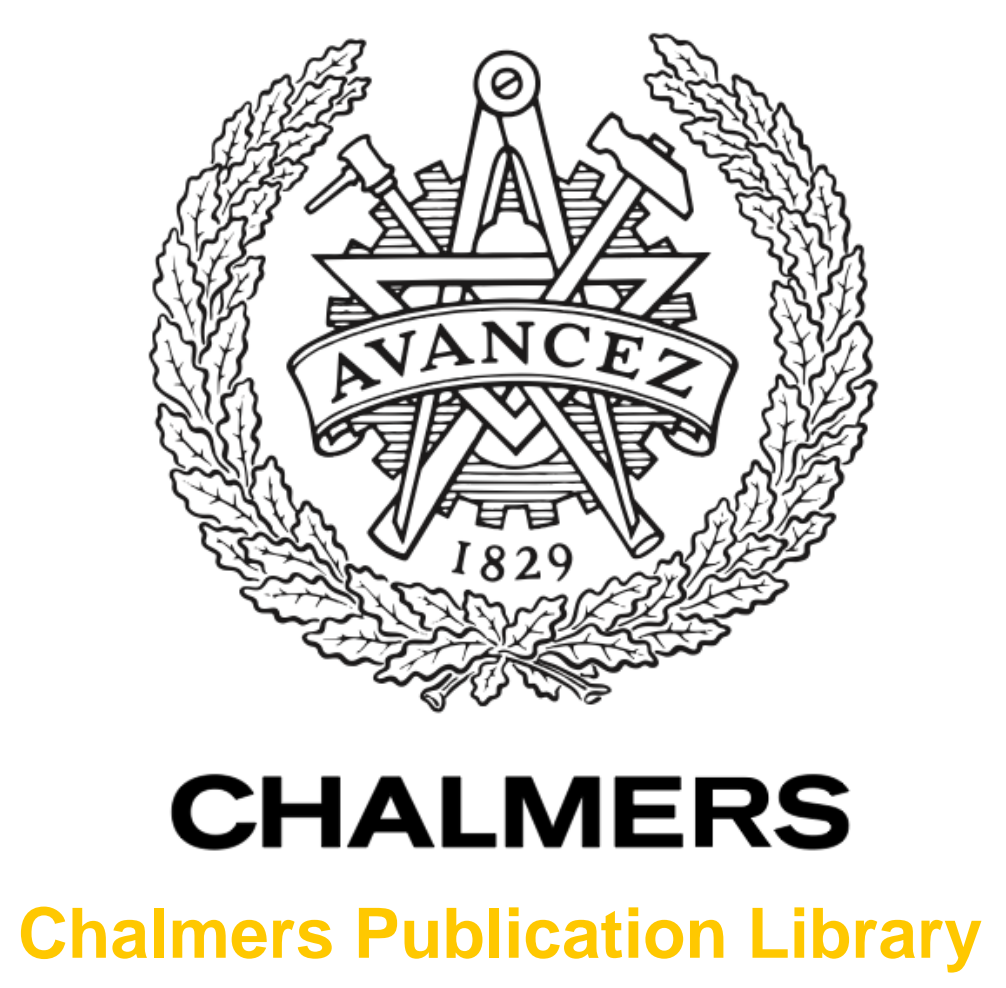

\title{
Contactless non-leaking waveguide flange realized by bed of nails for millimeter wave applications
}

This document has been downloaded from Chalmers Publication Library $(\mathrm{CPL})$. It is the author's version of a work that was accepted for publication in:

Proceedings of 6th European Conference on Antennas and Propagation, EuCAP 2012. Prague, 26-30 March 2012

Citation for the published paper:

Pucci, E. ; Kildal, P. (2012) "Contactless non-leaking waveguide flange realized by bed of nails for millimeter wave applications". Proceedings of 6th European Conference on Antennas and Propagation, EuCAP 2012. Prague, 26-30 March 2012 pp. 3533-3536.

http://dx.doi.org/10.1109/EuCAP.2012.6206199

Downloaded from: http://publications.lib.chalmers.se/publication/156664

Notice: Changes introduced as a result of publishing processes such as copy-editing and formatting may not be reflected in this document. For a definitive version of this work, please refer to the published source. Please note that access to the published version might require a subscription. 


\title{
Contactless Non-Leaking Waveguide Flange Realized by Bed of Nails for Millimeter Wave Applications
}

\author{
Elena Pucci and Per-Simon Kildal \\ Department of Signals and Systems \\ Chalmers University of Technology \\ Gothenburg, Sweden \\ elena.pucci@ chalmers.se, per-simon.kildal @ chalmers.se
}

\begin{abstract}
Waveguide flanges are typically used to connect and measure high frequency circuits. When good conductive contact is not provided between the joining flange surfaces, currents will flow between them, and thereby causing leakage and losses affecting the circuit performance. This work presents a nonleaking contactless waveguide flange made with bed of nails. The flange does not need any contact when connected to another smooth flange, since the pins surface and the smooth surface together form a stopband suppressing any current and wave propagation between the two joining surfaces of the flanges.
\end{abstract}

Keywords-waveguide flange; bed of nails; gap waveguide.

\section{INTRODUCTION}

Recently, a new waveguide created in the gap between contactless parallel metal plates has been presented and called gap waveguide [1]. The lower plate can be made with a ridge or groove or microstrip [2], surrounded by a bed of nails, which is a textured surface made of metal pins [3]. The pins work as an artificial magnetic conductor, stopping the field from propagating in any directions outside the ridge (or groove) with a bandwidth of this stopband determined by the type and dimensions of the periodic elements [4]. Gap waveguide circuits have been realized in [5] and [6]. The main advantage compared to other standard technologies, such as waveguides and microstrip transmission lines, is that it does not require metal contact between the split blocks. Additionally, it can be made of only metal and it has low loss [7]. being a promising candidate for applications at millimeter waves [8]. Waveguide flanges are typically used as transitions for measurements at millimeter and sub-millimeter waves. At these frequencies, high precision waveguide machining is needed in order to assure good alignment between the waveguide flanges [9]. Furthermore, mismatch and leakage can be generated if a proper electrical contact is not provided between the blocks. Fig. 1 presents the simulated S parameters of two connected waveguides WR 3. A standard waveguide WR 3 with flanges is shown in Fig. 2. When a gap of $20 \mathrm{um}$ is allowed between the connected waveguide flanges, the mismatch increases considerably compared to the desired case of no gap. With a gap of $40 \mathrm{um}$ the return loss reaches a $-10 \mathrm{~dB}$ level. First gap waveguide prototypes realized in MEMS technology at $280 \mathrm{GHz}$ were shown in [8]. However, the measurements of these prototypes were not successful due to a poor electrical contact between the waveguide transition, used for the measurement setup, and the micromachined silicon chips. This problem raised, therefore, the need of having new waveguide transitions at high frequency which perform well even when no good contact can be assured between the waveguide block and the circuit under test.

This paper presents a new waveguide flange realized by bed of nails and working between 190 and $320 \mathrm{GHz}$. This modified flange does not require conductive contacts when connected to another standard waveguide, thus avoiding all the related problems addressed during manufacturing process, which typically affect the performance of such flanges. It is also much easier to mount, because the flange screws do not need to be tightened very well or ensure good electrical contact like with standard flanges.

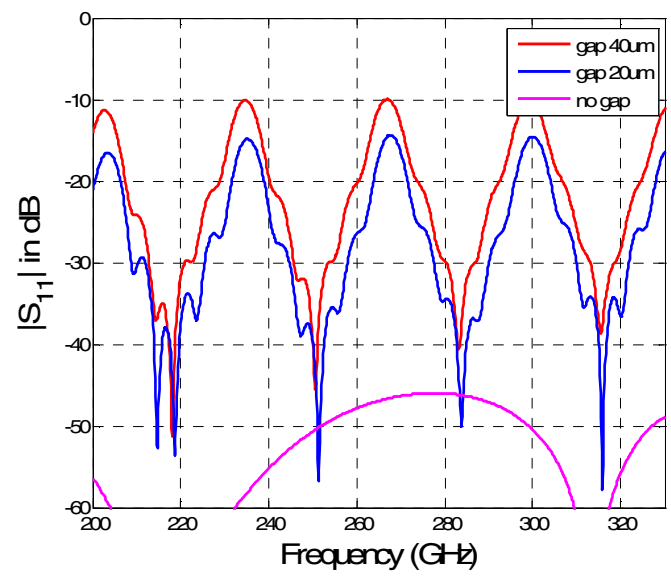

Figure 1. Simulated return loss for the WR 3 waveguide when a gap of 20 $\mathrm{um}$ and $40 \mathrm{um}$ is allowed between the flanges and when there is no gap between them.

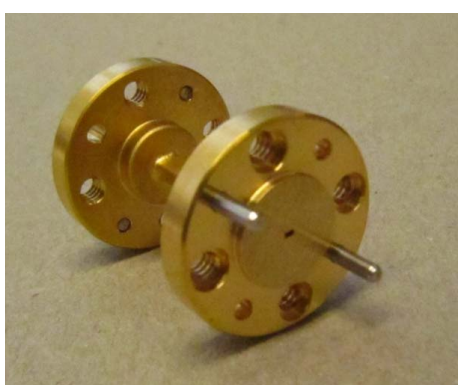

Figure 2. WR 3 wavaguide flanges.

This work has been supported by the Swedish Research Council VR. 


\section{STOP-BAND}

The operation frequency chosen is the same as the one of the waveguide WR 3, i.e., 220-325 GHz. Hence, the pins are designed for this frequency range, with the following dimensions, according to Fig. 3: $a=167$ um, $d=277.7$ um and $p=194 \mathrm{um}$. The pins provide a stopband from $180 \mathrm{GHz}$ to $400 \mathrm{GHz}$, as it can be seen from the dispersion diagram computed with CST Microwave Studio and shown in Fig. 4. The computation is done for an infinite unit cell made of a pin, separated from the upper PEC condition by an air gap of 56 um.

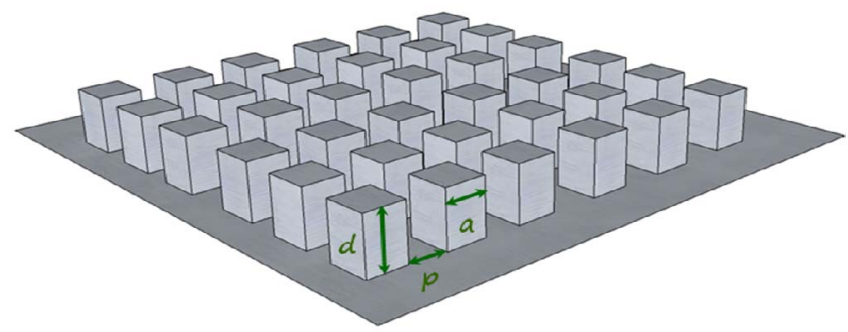

Figure 3. Sketch of lid of pins. Pins dimensions are $a=167 \mathrm{um}$ and $d=$ $277.7 \mathrm{um}$, and their distance is $p=194 \mathrm{um}$.

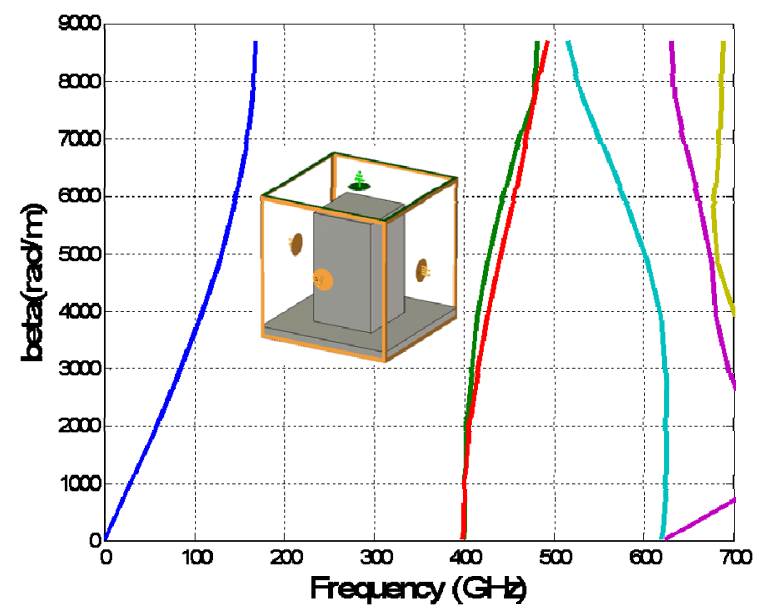

Figure 4. Dispersion diagram of an infinite unite cell made of a pin, separated from the upper PEC condition by an air gap of $56 \mathrm{um}$.

\section{DESIGN OF THE FLANGE}

The proposed solution is presented in Fig.5 (a). A standard WR 3 waveguide flange (shown in the photo) is modified by adding 2 rows of pins, mounted around the waveguide. In addition, there are four smooth metal surfaces of the same height as the pins, on each side of the waveguide cross section, before the first pin row. The two metal surfaces on either long side of the waveguide are quarter wavelength long to form, together with the smooth opposite flange, an impedance transformer that transforms an open circuit to a short circuit, thus avoiding reflections which can be created at the interface between the waveguide flanges. The pins create a parallel plate cut-off region in the air gap (Fig. 5 (b)) between the smooth and the textured flange, thus allowing the energy to propagate only along the waveguide without leaking out in the gap between the two flange parts. In order to create the stop-band region, the air gap must be smaller than quarter wavelength and the pin lengths typically approximately quarter wavelength [4]. In this case the air gap is chosen to be $56 \mathrm{um}$.

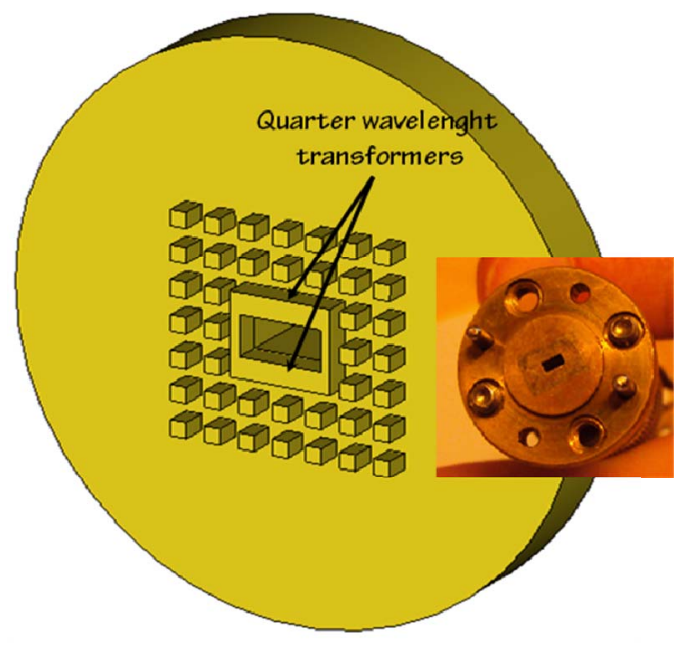

(a)

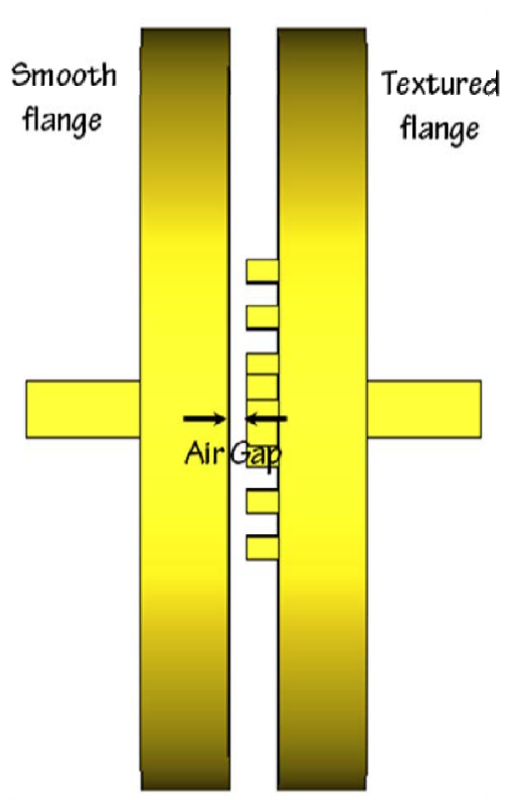

(b)

Figure 5. (a) Design of the waveguide flange with pins beside a photo of standard WR3 flange. (b) Side view of the textured flange with pins when it is connected to a smooth flange. The air gap between the flanges is $56 \mathrm{um}$.

\section{SimULATION RESULTS}

In this section simulation results are presented in terms of Sparameters for the case presented in Fig. 5 (b), where the flange modified with pins is connected to another standard one. We consider now this simple case in order to reduce the 
computation time but it must be pointed out that the smooth flange can also be replace by any other circuit.

A parametric study is also performed to check how the results vary by changing few parameters. Firstly, we studied the effect of the solid walls added at the shorter sides of the waveguide. These lateral surfaces do not need to be quarter wavelength long, therefore we can vary their width as shown in the plot in Fig. 6. As it can be seen, by reducing the width $b_{1}$ the upper frequency of the bandwidth increases.

Even better results are obtained when the squared walls, surrounding the waveguide, are replaced by circular walls, as shown in Fig. 7, with return loss below $-20 \mathrm{~dB}$ between 180 and $320 \mathrm{GHz}$. This can be explained by noticing that the length $a_{1}$ of the smooth surfaces must be $\lambda_{\mathrm{g}} / 4$ long to transform the open circuit in a short circuit. By gradually reducing $a_{1}$, using a circular surface instead of a squared one, the frequency increases, thus increasing the bandwidth.

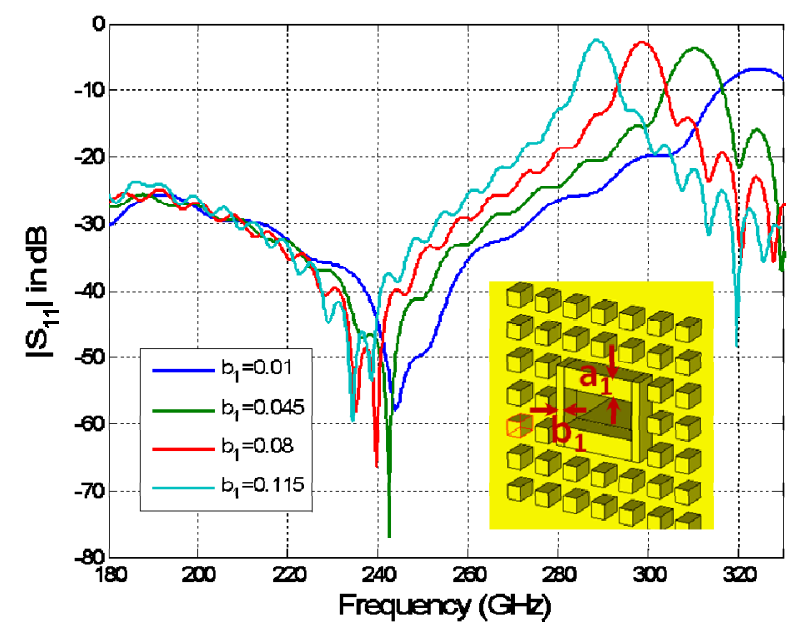

Figure 6. Simulated reflection coefficient for different widths $b_{1}$.

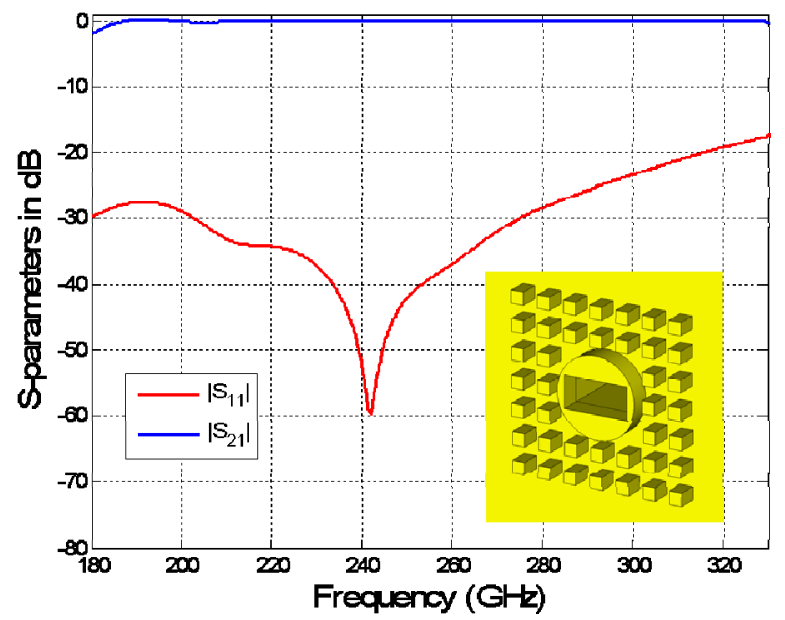

Figure 7. Simulated S-parameters when the walls surrounding the waveguide are circular.

\section{COMPARISON WITH CHOKE FLANGE}

It is of interest to compare the proposed design with already known choke flanges [10, p. 131]. The principle of operation is similar because the choke is a $\lambda_{\mathrm{g}} / 4$ deep ring engraved in the flange, at a distance $\lambda_{\mathrm{g}} / 4$ from the waveguide. Fig. 8 shows how both solutions work well and in good agreement. They can be both easily realized by machining. The lid of pins offers, however, more degree of freedom in the choice of the position and application. In this work we showed how they can be used to connect two waveguides, by inserting them on one of the flanges. They could also be used to combine two pieces of waveguide along their longitudinal walls, for example, avoiding flow of currents at the joints and leakage when a gap is present between them. Furthermore, the pins surface is isotropic, working as high impedance surface for both polarizations, compared to the choke, which is basically made with a corrugation corresponding to a soft surface.

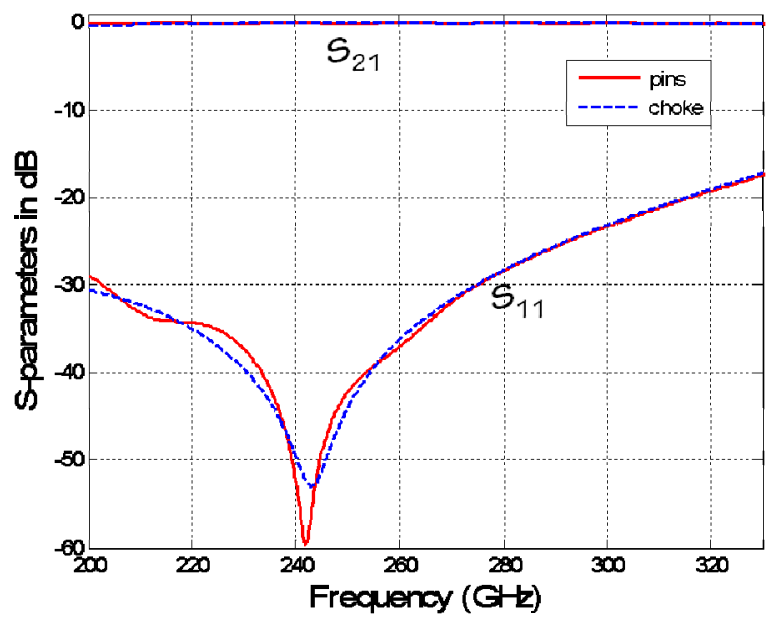

Figure 8. Simulated S-parameters for the waveguide with textured flange (red curve) and for the choke flange (dashed blue curve).

\section{CONCLUSIONS}

This paper showed how the pins surface, used to make gap waveguides, can be used on one face of a standard waveguide flange to avoid power loss and mismatch that are often experienced when combining waveguide circuits at high frequency. Simulation results showed that the textured flange produces a return loss below $-20 \mathrm{~dB}$ between 190 and 320 $\mathrm{GHz}$, being totally contactless to the other smooth flange. For practical reasons the flange should have some ridge or pins working as spacers between the two surfaces, located near the rim of the flange, in order to make a well defined airgap between the two surfaces. The airgap was in the simulations assumed to be constant equal to $56 \mathrm{um}$. These spacers have not been shown in the drawings, and will have no effect on resulting performance. The result is assured as far as the air gap, separating the two flanges, is smaller than quarter wavelength, allowing the pins to work as a high impedance 
surface. The proposed solution works as well as already known choke flanges and it can be also used to connect waveguide spilt blocks and to measure dual polarizations without need of good electrical contacts between the combined parts.

\section{ACKNOWLEDGMENT}

The authors would like to thank the MEMS group at the MC2 Department, Chalmers University of Technology, for their good feedback on understanding manufacturing issues related to high frequency circuits.

\section{REFERENCES}

[1] P.-S. Kildal, E. Alfonso, A. Valero-Nogueira, E. Rajo-Iglesias, "Local Metamaterial-Based Waveguides in Gaps Between Parallel Metal Plates," IEEE Antennas and Wireless Propagation Letters, vol.8, pp.84$87,2009$.

[2] P.-S. Kildal, "Three metamaterial-based gap waveguides between parallel metal plates for $\mathrm{mm} /$ submm waves", 3rd European Conference on Antennas and Propagation, 2009. EuCAP 2009. Berlin, Germany, 2327 March 2009.

[3] M. G. Silveirinha, C. A. Fernandes, and J. R. Costa, "Electromagnetic characterization of textured surfaces formed by metallic pins," IEEE Transactions on Antennas and Propagation, vol. 56, no. 2, pp. 405-415, 2008 .
[4] E. Rajo-Iglesias, P.-S. Kildal, "Numerical studies of bandwidth of parallel plate cut-off realized by bed of nails, corrugations and mushroom-type EBG for use in gap waveguides", IET Microwaves, Antennas \& Propagation, Vol. 5, No 3, pp. 282-289, March 2011.

[5] E. Rajo-Iglesias, A. Uz Zaman, P.-S. Kildal, "Parallel plate cavity mode suppression in microstrip circuit packages using a lid of nails", IEEE Microwave and Wireless Components Letters, Vol. 20, No. 1, pp. 31-33, Dec. 2009.

[6] P. S. Kildal, A. U. Zaman, E. Rajo-Iglesias, E. Alfonso, and A. ValeroNogueira, "Design and experimental verification of ridge gap waveguide in bed of nails for parallel-plate mode suppression", IET Microwaves, Antennas \& Propagation, vol. 5, no. 3, pp. 262-270, 2011.

[7] E. Pucci, A. U. Zaman, E. Rajo-Iglesias, P.-S. Kildal and A. Kishk, "Study of Q-Factors of Ridge and Groove Gap Waveguide Resonators", submitted to IEEE Transactions on Microwave Theory and Techniques, June 2011.

[8] S. Rahiminejad, A. U. Zaman, E. Pucci, H. Raza, V. Vassilev, S. Haasl, P. Lundgren, P.-S. Kildal and P. Enoksson, "Micromachined ridge gap waveguide for sub millimeter and millimeter wave applications", accepted for publication to Sensors and Actuators A: Physical, an international open access journal by Elsevier, 2012.

[9] C. Oleson, A. Denning, "Millimeter Wave Vector Analysis Calibration and Measurement Problems Caused by Common Waveguide Irregularities," ARFTG Conference Digest-Fall, 56th, vol.38, no., pp.19, Nov. 2000

[10] D.M. Pozar, Microwave Engineering, 2nd ed., pp. 131-132, John Wiley, New York, 1997. 